\title{
ANIMAL HEALTH RESEARCH \\ AT A TIME OF NATIONAL SECURITY: THEN WHAT?
}

\author{
Mark R. Ackermann \\ Professor of Veterinary Pathology \\ lowa State University of Science and Technology
}

\section{Introduction}

The biological relationship between animals and humans has never been more intertwined. The persistent outbreaks of diseases such as Escherichia coli O157:H7, the malicious spreading of anthrax spores, the emergence of West Nile Virus, and the economic impact of non-zoonotic diseases such as Foot and Mouth Disease, have gripped the attention and, in a peculiar way, the lure of the general public and scientific community (Table 1). These events, coupled with the plethora of other animal diseases that range from cancer to mastitis, have triggered introspection of animal health as it relates to human health, national security, and the national economy. Clearly, there are a number of medical diseases of animals, both infectious and non-infectious, that are vital to: 1) the basic understanding of disease processes that overlap in human and veterinary medicine; 2) the general health and well-being of animals (and potentially humans); and 3) the national economy and bioterrorism. In addition, veterinarians, physicians and scientists have long been aware of the many nearly identical similarities between diseases and conditions of animals to those of humans. However, animal health issues have been strongly tied with issues of agriculture such as soil science, plant health, food stamps, farm management, waste disposal, etc. This tie, in certain ways, has obscured the view of veterinary medicine and animal health as a close correlate to human medicine and health. Is it time for aspects of veterinary medicine and animal health to be viewed, sideby-side, with human medicine and health?

Table 1. Selected zoonotic (passed from animals to humans) diseases with potential for human outbreaks and biosecurity risk and selected non-zoonotic diseases (diseases not passed to humans) of potential economic importance.

\section{Zoonotic diseases:}

anthrax, Yersinia pestis (bubonic plague), Francisella tularensis (tularemia), brucellosis, leptospirosis, rabies, tuberculosis, Nipahvirus, Hendravirus, Chlamydia, bovine spongioform encephalopathy (BSE; Mad cow), food and water-borne diseases: E. coli 0157:H7, west nile virus, VEE, EEE, WEE, Giardia, Salmonella; viral hemorrhage fevers: Marburg, hantavirus.

Non-zoonotic diseases:

Foot and Mouth disease (FMD)*, African swine fever, Vesicular exanthema, vesicular stomatitis, Mycoplasma mycoides (contagious pleuropneumonia), rinderpest, classical swine fever (Hog Cholera).

\footnotetext{
${ }^{*}$ Can infect humans on rare occasions
} 
Some diseases of veterinary importance have been scarcely known in previous times, but their importance has skyrocketed due to outbreaks and/or newly discovered attributes that are now recognized in diseases of humans. Scrapie, for example, was a little known disease entity of sheep and considered an oddity nearly 50 years ago until Dr. William Hadlow, a veterinary pathologist, linked similar aspects of Scrapie in sheep with those of Kuru in humans. ${ }^{1-3}$ Today, it is becoming increasingly clear that the Scrapie disease agent has important similarities to bovine spongioform encephalopathy (BSE; a.k.a., Mad Cow disease) in cattle which, in turn, is linked by some investigators to the emergence of a new and deadly variant of Creutzfeld-Jacob Disease in humans (Fig. 1). ${ }^{2}$ Similarly, E. coli O157:H7 and strains with similar attributes were first detected in animals and considered relatively unimportant in animals, but have since risen in prominence as important food pathogens that can cause diarrhea, anemia, and life-threatening renal disease in children and susceptible adults. ${ }^{4,5}$ These are but two examples of the types of animal diseases that were once considered incidental diseases with no relation to human health but now highlight and underscore the blending of animal and human disease.

Figure 1. A little known disease of sheep becomes well known in human medicine. The similarities of Scrapie in sheep with Kuru in humans were first suggested by William Hadlow. Thereafter, cattle fed sheep tissues developed bovine spongioform encephalopathy (BSE) and a new variant of Creutzfelt-Jacob disease was identified in humans.

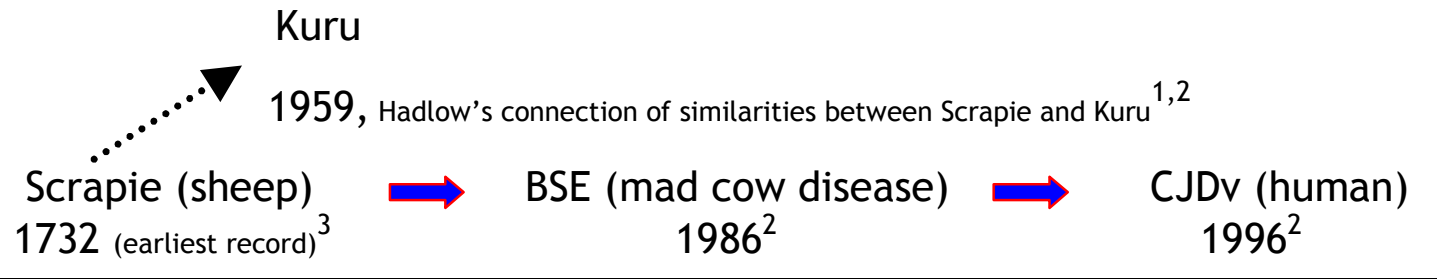

General Health and Well-Being of Animals (and potentially humans)

Other animal health issues seemingly have, at this time, no connection with human health and biosecurity; however, they are important to the general health and well being of animals because of the suffering they inflict and the economic loss they impose on producers. For example, pneumonia, certain forms of diarrhea, and mastitis cost billions in production loss each year, not to mention the animal suffering that they invoke. Research on such diseases could be eliminated if money is shifted to bioterrorism, biosecurity, and food safety. Unfortunately, loss of research activity in these diseases is shortsighted and somewhat risky because some could become even more widespread problems in animals, and some, like Scrapie and E. coli O157:H7, could eventually contribute to human disease. 
A better understanding of some of these diseases may also provide a fuller appreciation of the pathogenesis of human disease. Respiratory syncytial virus of cattle and sheep, for example, are very similar to their human counterpart and make excellent animal models. Spontaneous non-infectious and aging diseases of dogs (osteosarcoma, prostatic cancer, hemangiosarcoma, hyperlipidemia), cats (type II diabetes mellitus, lymphoma), non-human primates, and other species are numerous and have many potential similarities to their counterparts in humans. In fact, cancer in domestic animals may prove to parallel the incident of cancer in humans in some instances. However, many such diseases remain under-investigated.

In addition, xenotransplantation of organs from pigs to humans will require close scrutiny of several viruses of pigs (such as the porcine retrovirus, circovirus, and hepatitis E virus) for their potential to replicate in human recipients. Wildlife diseases such as chronic wasting disease of deer and elk, tuberculosis of deer, and brucellosis of elk and bison, are spreading and all have human health implications. Rodents and other animals used for research, and racing animals used for entertainment also have their own set of diseases and conditions that may affect human health. Who, for example, wants data from laboratory rats or mice that spontaneously grow tumors or carry an infectious agent? In short, a variety of diseases that appear to be only of veterinary importance today have potential in fitting in the mission of the United States Department of Health and Human Services (DHHS), because investigative research of these diseases may: 1) increase understanding of the comparative aspects of disease pathogenesis, and 2) lead to prevention or therapeutic strategies against diseases and conditions of humans in the future.

\section{National Economy and Bioterrorism}

Bioterrorism can result in death, inflict immense human suffering, and/or greatly alter the national economy. Recent bioterrorism threats coupled with food safety and security issues have greatly influenced research priorities. Simultaneously, Americans seem less and less worried about having a plentiful supply of food from animals, because meat and dairy production in the United States generally meet demand and food is relatively inexpensive. Such changes in priorities, coupled with limitations in USDA funding of animal health issues, may result in subtle, but continual shifts away from research activity on animal diseases with no immediate threat listed above (pneumonia, diarrhea, etc.). What would be lost may include insight of the comparative features of animal disease as they relate to similar human diseases. In addition, it would erode information on animal diseases that are currently unforeseen threats to human health in the future. 
The National Institute of Animal Health (NIAH)

Although it will likely never happen, aspects of animal health research could be more closely aligned with human diseases as an institute within the National Institutes of Health $(\mathrm{NIH})$. The $\mathrm{NIH}$ already houses institutes for nursing, mental health, dental health and has numerous specialized centers for many diseases and conditions of humans. An agency with a mission and focus on animal health could be named the National Institute of Animal Health (NIAH) and exist along with the National Institute of Allergy and Infectious Diseases (NIAID), the National Heart, Lung, and Blood Institute (NHLBI), and other Institutes and Centers. Potentially, the NIAH could strike a balance in the funding and resources that would be allocated to outbreaks and crisis issues and those areas that are not an immediate threat to humans, but require a long-term appropriation. If expanded, the NIAH could also serve under-represented areas such as wildlife and aquaculture and perhaps have a regulatory role to oversee care and use of animals used for research.

Divisions of the NIAH

What would the NIAH look like? It could house six divisions that are primarily focused on extramural research funding and a few divisions with regulatory activity (Table 2). A first division could emphasize the interaction of animal health on human health. This would include supporting the development of animal models of human disease, pathogens of bioterrorism, and the maintenance of healthy animals for research. The second could emphasize animal health research simply for animal health, with an emphasis on foodproducing animals and aquaculture. The third could include animal diseases to be kept out of the United States, both zoonotic and non-zoonotic, and also include emerging animal diseases throughout the world. The fourth could be for wildlife and environmental habitat, particularly for the control of existing wildlife infectious diseases with potential human health concerns, and eventually, the

Table 2. Divisions of The National Institute of Animal Health (NIAH), a proposed new institute in the National Institutes of Health (NIH).

Division 1: Zoonotic disease, food safety, bioterrorism, animal models of human disease, regulatory manners of mice, rats, non-human primates.

Division 2: Diseases of food animals. Cattle, sheep, swine, poultry, aquaculture.

Division 3: Foreign animal diseases of national security/emerging diseases of animals.

Division 4: Wildlife diseases and environmental habitat.

Division 5: Racing animals (horses, dogs), domesticated pets (dogs, cats, horse), animal shelters, the health benefits of human-animal bonds.

Division 6: Training programs for animal health care specialists. 
elimination of such diseases. The fifth could emphasize disease and safety for racing animals and domestic pets, operation of animal shelters, and the psychological benefits of the human-animal "bond." The sixth and last division could emphasize training programs for animal health specialists. All $\mathrm{NIH}$-funded projects have requirements for adequate animal care; therefore, the continual training of such experts is vital.

Food Safety, Animal Nutrition, Environment, and Aquatic Life

At least two areas may not fit within such an institute or would require overlap with agriculture: food safety and animal nutrition. It may be reasonable that pre-harvest (pre-slaughter) food safety issues could be funded under the $\mathrm{NIAH}$ and post-harvest (post-slaughter) issues could remain under the USDA. For nutrition, perhaps animal nutrition studies for animals would remain in the USDA. In addition, there may be overlap with environmental agencies concerned with animal waste issues and the effects of water pollution on aquaculture and marine life. Fresh-water and marine life are often important sentinels for environmental pollution and disease outbreaks or accumulations of toxins within fish and other aquatic life can be critical indicators of environmental pollution. Details in deciphering these issues would be, admittedly, numerous.

\section{Summary}

Many microbiological agents that infect animals as well as many other aging and metabolic diseases of animals are often similar to correlates of diseases in humans. In the past, veterinary medicine and animal health have been aligned closely with agriculture and this structure may maintain a subtle barrier for appreciating the similarities of animal and human diseases and conditions. Although some animal diseases and conditions may not have a direct connection with human disease and health, many of these cause great suffering in animals and, in addition, historical events have repeatedly demonstrated that very obscure diseases of animals can eventually affect human health in a direct manner. Therefore, maintaining and even expanding research on animal diseases and conditions is prudent for human health, and it is economically savvy. Aligning aspects of veterinary medicine and animal health with the mission of the Department of Health and Human Services (DHHS) and the National Institutes of Health $(\mathrm{NIH})$, may be beneficial in revealing subtle connections to human disease processes. Identification of such connections may enhance disease prevention and treatment in both animals and humans. 


\section{Endnotes}

1. Hadlow, William J. (1959). Lancet ii, 289.

2. Hadlow, William J. (1999). Reflections on the transmissible spongioform encephalopathies. Veterinary Pathology, 36, 523-529.

3. M' Gowan, J.P. (1914). Investigation into the disease of sheep called "Scrapie." Edinburgh: William Blackwood and Sons.

4. Moon, Harley L., personal communication.

5. Whipp, S.C., Rasmussen, M.A., and Cray, W.C. (1994). Animals as a source of Escherichia coli pathogenic for human beings. Journal of the American Veterinary Medical Association, 204, 1168-1175. 